\title{
Le devenir paysager. De quelques paysages postindustriels entre arts visuels et poésie
}

\author{
ÉLISE LEPAGE
}

This essay proposes an analysis of the concept of landscape in contemporary Quebec visual arts and poetry. A brief archaeology of the landscape shows the pictorial origins of this genre, as well as the anthropocentrism from which it proceeds. Indeed, when the landscape becomes an autonomous form during the Renaissance, it is assumed that the subject necessarily pre-exists the landscape. The landscape is thus considered one representation among others of reality. The importance of this human-centred landscape in contemporary arts will be studied in Isabelle Hayeur's photographs and videos, as well as in the poetry of Pierre Nepveu, Louis-Jean Thibault, Robert Melançon, and Claude Paradis. If the subject remains central to their representations, these artists also offer other critical approaches. More than a mere aesthetic motif or a touristic cliché, the landscape is becoming an ethical and aesthetic meeting place, implying new relationships between the landscape and the subject, yet to be explored.

Cet article propose une réflexion sur le concept de paysage dans les arts visuels et la poésie québécoise contemporaine. Une brève archéologie du paysage permet de souligner ses origines picturales ainsi que l'anthropocentrisme dont il procède. En effet, lors de son émergence à la Renaissance, on considère que le sujet préexiste au paysage qui n'est que l'une de ses représentations du réel, auquel il donne forme. L'étude de plusieurs séries photographiques et vidéos d'Isabelle Hayeur et de quelques recueils de poèmes publiés depuis 2000 par Pierre Nepveu, Louis-Jean Thibault, Robert Melançon et Claude Paradis permet de montrer la prégnance du paysage dans les arts aujourd'hui. Si la subjectivité demeure centrale dans leurs représentations paysagères, celles-ci proposent d'autres perspectives d'analyse. Plus que simple motif esthétique ou cliché touristique, le paysage est en voie de devenir un lieu de rencontre, de convergence éthique et esthétique. Quels peuvent donc être alors les nouvelles relations entre paysage et subjectivité? 
L

a présente réflexion prend la forme d'un diptyque paysager qui propose de mettre en regard quelques œuvres de l'artiste visuelle Isabelle Hayeur avec quelques recueils de poèmes publiés Jepuis 2000. Ce dialogue entre objets visuels - il s'agira de photographies et de vidéos - et objets textuels ne se justifie pas par un simple désir d'exotisme qui pousserait à aller voir ce qui se fait dans d'autres langages; il est au contraire presque rendu nécessaire de par le sujet que l'on se propose. Le paysage est en effet un concept d'origine pictural et il convient à cet égard de prêter attention à la mémoire qu’entretiennent les poèmes de cette généalogie esthétique. De surcroît, il n'est pas exagéré de dire qu'avant la littérature (et particulièrement la poésie), plusieurs langages artistiques ont entrepris de mettre en question ce concept - que l'on songe au land art, aux installations in situ, à l'architecture ou encore à la photographie et à la vidéo.

Les paysages autour de nous ne cessent de changer et - cause ou conséquence - notre regard sur eux change souvent de manière aussi spectaculaire. La ville vient en premier lieu à l'esprit : espace en continuelle transformation, elle est toujours bariolée de zones de démolition, de construction, de changement de plan de circulation et de zonage. « Habiter en ville », « habiter la ville » peut désigner des réalités bien différentes selon la taille de l'agglomération, selon l'époque et les lieux fréquentés au quotidien. La ville change nos façons de l'habiter, de l'imaginer et de la dire. Les constantes et nombreuses mutations de l'espace urbain sont ainsi autant d'invitations pour artistes et poètes à explorer de nouveaux (mi)lieux urbains et à poser un regard interrogateur sur ces paysages inédits qu'ils créent et qui appellent de nouvelles façons de les investir. La présente réflexion vise à étudier ces paysages en constante transformation et leurs représentations contemporaines à travers l'œuvre plastique d'Isabelle Hayeur et quelques récents recueils poétiques. L'œuvre de premier plan d'Isabelle Hayeur, remarquable de par son originalité et sa variété de langages esthétiques, a fait partie d'expositions majeures au Québec et est exposée régulièrement partout au Canada, mais aussi aux États-Unis et en Europe. On l'a donc retenue autant pour son éminence que son exemplarité sur le sujet. Quant aux recueils de poèmes, Lignes aériennes (2002) de Pierre Nepveu, Reculez falaise (2007) de Louis-Jean Thibault, Le paradis des apparences (2004) de Robert Melançon et Carnet d'un improbable été (2013) de Claude Paradis, ils ont été choisis car, chacun à leur façon, ils problématisent le paysage et entrent ainsi en résonance avec l'œuvre de Hayeur. Cette approche intermédiale permet de faire dialoguer plusieurs langages esthétiques (poésie, photographie et vidéo dans le cas présent) sur les paysages contemporains. L'objectif consiste à voir comment ces différents langages interrogent la fabrication des paysages qui s'offrent à nos regards et constituent la toile de fond sur laquelle nos habitus prennent forme.

On rappellera dans un premier temps quelques prémisses théoriques touchant à l'histoire et aux théories du paysage, avant de présenter les principaux aspects de la pratique d'Isabelle Hayeur. Ses montages photographiques créent des paysages inédits, tandis que d'autres photographies et vidéos donnent à voir l'irréalité de la banlieue nord-américaine. Cette étude des productions visuelles d'Hayeur invitera à repenser la place qu'occupe le sujet dans l'élaboration du paysage, question que l'on transposera ensuite aux recueils de poèmes (la relation entre sujet et paysage), en montrant l'importance de la préoccupation paysagère dans la poésie québécoise contemporaine. Cette dernière se tourne alternativement vers le village et ses 
dernières réinventions ou vers le monde urbain, mais en s'attardant alors sur ses périphéries, ses ruelles et arrière-cours, ses envers de décor et autres banlieues résidentielles et commerciales, paysages familiers de l'ère postindustrielle qui est la nôtre. Il convient de restituer au paysage sa dimension temporelle et par-là même sa variabilité ${ }^{1}$, et en fin de compte de réévaluer la valeur accordée à la subjectivité; d’où l'importance du concept imperfectif de « devenir » dans notre approche...

\section{Le paysage, côté pictural}

Le mot « paysage » est à l'origine un concept pictural datant du milieu du XVI siècle et qui fait son entrée dans les dictionnaires au cours du XVII ${ }^{e}$ siècle. Il est d'intérêt de souligner que durant l'Antiquité et le Moyen-Âge, aucune civilisation n'est arrivée à un terme pour ce concept. Dans L'invention du paysage [I989] (2000), Anne Cauquelin montre que les Grecs distinguaient le topos - le site, le lieu physique où l'on se trouve et que les cartographes représentent par un point ou une zone sur une carte - de la chôra. La chôra, tout comme le topos, se traduit aujourd'hui par le mot «lieu », même si elle se rapproche davantage du concept de paysage. La chôra désigne en effet le lieu existentiel qui tient compte de toutes les particularités qui le rendent unique. Elle porte les traces et les stigmates de ceux qui l'habitent, mais en retour elle les façonne aussi, marquant ainsi leur appartenance à ce milieu. Tout comme le topos, la chôra se traduit aussi par « lieu », mais désigne plutôt l'appartenance d'un corps (objet ou sujet) à un milieu. Dans son ouvrage Écoumène (2000), Augustin Berque lui consacre de belles pages, soulignant le fait qu'elle est à la fois « empreinte et matrice » (22) des êtres. Ce terme est celui qui semble le plus proche du concept de « paysage », mais peut-être est-il encore préférable de traduire chôra par « milieu ». Comme l'établit Anne Cauquelin, « [i]l n'y a, chez les Grecs anciens, ni mot ni chose ressemblant de près ou de loin à ce que nous appelons « paysage » » $(2000,25)$. Certes, les Grecs étaient conscients de la nature qui les entourait, mais « [s]ans doute la Nature ne se figurait-elle pas dans la forme du paysage. Si elle acceptait d'être figurée concrètement, c'était en terme d'ordonnancement, de distribution ordonnée » (Cauquelin 2000, 26)². Il faut donc attendre beaucoup plus tard, le début des temps modernes, pour que le paysage s'introduise par la fenêtre de certains tableaux de la Renaissance jusqu’à prendre toute la place sur la toile. Le Thresor de la langue francoyse de Jean Nicot le mentionne dès I606 comme « mot commun entre les paintres », attestant donc que ce sont bien les premières représentations de paysage - notamment dans la peinture hollandaise du XVI ${ }^{\mathrm{e}}$ siècle - qui ont créé le mot. À la fin du siècle, la première édition du Dictionnaire de l’Académie française (i694) marque déjà la dissémination du terme. Le « païsage » est alors une « estenduë de païs que l'on voit d'un seul aspect », mais « [i]l signifie aussi un tableau qui represente un païsage » (Dictionnaire de l'Académie). Cette double acception témoigne de l'ambiguité du concept, employé tantôt pour parler du référent («l'étendue de pays »), tantôt pour évoquer les représentations artistiques qui en sont faites (« un tableau qui représente un paysage »). Cette ambiguité est d'autant plus problématique qu'elle est allée en s'amplifiant avec le temps, le terme jouissant d'une importante inflation sémantique; on parle en effet aujourd'hui de paysage industriel, paysage politique, paysage audiovisuel, paysage virtuel, sans oublier les parcs ou aménagements « paysagés ». Dans le cadre de cette réflexion, nous envisagerons le terme de « paysage » dans son sens le plus généreux, le plus vaste, sans le restreindre aux seuls paysages « naturels », et ce, parce que tout paysage n'est jamais purement naturel au sens où Rousseau parlait d'un «état de nature ». 
Comme le laisse entendre la première définition du Dictionnaire de l'Académie française citée plus tôt, un paysage est une étendue de pays qui peut être saisie en un regard, sans avoir besoin de se déplacer ni même de tourner la tête. Ce premier constat présuppose donc nécessairement la présence, ici hors cadre, d'un sujet capable d'embrasser du regard cette étendue de pays. «Quand je découvre un paysage jusquelà caché par une colline, c'est alors seulement qu'il devient paysage », écrit Maurice Merleau-Ponty dans Sens et non-sens [I948] (I966), avant de poursuivre : « Ce monde qui avait l'air d'être sans moi [...] c'est moi qui le fais être. » (5I). Une subjectivité préexiste nécessairement à toute conscience paysagère dans la mesure où elle effectue - de façon plus ou moins concertée - un travail de découpage, d'agencement d'éléments réels pour produire une représentation visuelle plus ou moins artificielle. Comme l'écrit Alain Roger dans son Court traité du paysage (1997), « un paysage n'est jamais naturel, mais toujours culturel » (I28) puisqu'il est le fruit d'une composition qui porte la marque culturelle du sujet qui l'élabore. Roger souligne à quel point « L'art [...] artialise le pays en paysage » (2I), c'est-à-dire que les représentations artistiques ou culturelles que nous connaissons éduquent notre œil et nous apprennent à voir le réel à la façon de tel ou tel artiste. Anne Cauquelin (2009) ne dit pas autre chose lorsqu'elle écrit que « [...] les peintres nous ont appris à voir du paysage là où nous ne verrions rien ou un simple fouillis. [...] [C]ette forme paysagère agit sur nos perceptions du monde comme forme a priori qui trace le cadre de ce que nous sommes à même d'appréhender » (xviii). Comme le formule Paul Klee, « l'art ne reproduit [plus] le visible, il rend visible» (cité par Lapierre I995, II4). Roger cite les exemples bien connus de lieux tels que la Sainte-Victoire qu'on connait bien souvent par les toiles de Cézanne avant même que de la voir en réalité ou encore le pont Mirabeau du poème d'Apollinaire. On pourrait multiplier des exemples tout aussi éclairants pour le Canada - pensons au pont Provencher dans l'œuvre de Gabrielle Roy - ou pour le Québec - les toiles de Clarence Gagnon ou de Jean-Paul Lemieux. Ce processus, qu’Alain Roger nomme l'artialisation - terme emprunté à Montaigne -, permet de démystifier le fameux paradoxe anti-platonicien d'Oscar Wilde selon lequel « la Nature imite l'Art, bien plus que l'Art imite la Nature ${ }^{3}$ ». Notons que si la présence du sujet est traditionnellement hors cadre, les artistes s'ingénient plus souvent qu'autrement à jouer avec sa position. On peut songer au cas où ce sujet est inclus dans l'œuvre, représentant ainsi soit l'artiste (l'auteur ou le peintre), soit l'esthète (le lecteur ou le spectateur) ${ }^{4}$.

Nous avons montré qu'à tout paysage préexistait une subjectivité; soulignons à présent que cette subjectivité va produire une représentation du réel. « Le paysage est le visage du pays », écrit Michel Deguy $(2005,2 \mathrm{I})$. Par-delà la belle densité de la formule, déplions ce qu'elle implique : le pays est le référent, concret, situable et mesurable de façon objective. Le paysage au contraire est une représentation subjective, changeante, culturelle du pays. À la rigueur, il faudrait parler du pays, réalité singulière et objective, et des paysages, mimèsis plurielles et subjectives. Le créateur des jardins d'Ermenonville, René-Louis Girardin, établissait déjà cette distinction : « Le long des grands chemins, et même dans les tableaux des artistes médiocres, on ne voit que du pays; mais un paysage, une scène poétique, est une situation choisie, ou créée par le goût et le sentiment » (I992, 55). C'est bien en cela qu'un paysage n'est jamais naturel; son thème, son motif peuvent l'être lorsque la fameuse « étendue de pays » est, selon divers topoï, campagne riante, 
montagne majestueuse, mer déchaînée ou île déserte; mais de par les dimensions subjective et technique qu'il implique, le paysage est au contraire une création artificielle dont l'agencement a été pensé et dont les composantes ont été choisies et savamment disposées.

Enfin, toute représentation implique nécessairement un travail de composition. La représentation paysagère résulte d'un découpage et d'un agencement plus ou moins artificiel du réel. Il n'est que de penser au photographe qui, en définissant l'étendue de champ de sa photographie, choisit d'inclure tel arbre situé à gauche et d'exclure tel pan de mur sur la droite. Il peut aussi choisir de se déplacer de quelques pas entre plusieurs prises de vue pour se placer à l'ombre ou en pleine lumière, changeant ainsi l'éclairage du cliché à venir, ou pour inclure une partie de l'étendue d'eau au premier plan ou modifier la proportion entre ciel et terre. Autrement dit, la configuration joue un rôle de premier plan en cela qu'elle va pour beaucoup contribuer aux lectures possibles du paysage produit : créer telle perspective, zone de lumière, effet de contraste ou rappel de couleur permet de guider le regard du spectateur. Car contrairement à une conception commune - et qui semble remonter jusqu'au dictionnaire de I694 de l'Académie française -, si la saisie d'un paysage peut être instantanée, sa lecture s'inscrit dans la durée. On déchiffre peu à peu les détails de l'ensemble au fil du cheminement de l'œil (dans une œuvre visuelle) ou de la lecture (en littérature) et on découvre la cohérence ou la dysphorie de l'ensemble au terme de ce parcours. Et cela sans compter les cas où l'on redécouvre un même paysage dans des circonstances différentes ou après un intervalle de temps. Il y aurait bien à dire sur cette dimension chronologique de la saisie du paysage et de ses lectures, de ses traversées, mais on se bornera ici à souligner que, quel que soit le langage esthétique dans lequel il prend forme, le paysage implique trois composantes essentielles à son élaboration et son appréciation : la présence d'une subjectivité, la fonction de représentation et le travail de composition.

\section{Les paysages d'Isabelle Hayeur}

Les artistes qui s'intéressent aux paysages font jouer ces trois composantes de différentes façons. Isabelle Hayeur est du nombre de ceux qui ont fait du paysage leur lieu d'investigation. Ses photographies et vidéos donnent à voir des paysages « qui paraissent «naturels», mais qui sont créés de toutes pièces », mettant ainsi en question « l'ambivalence de notre rapport au monde » à travers une pratique artistique qu’elle qualifie de « politique et poétique » (Hayeur5). Artiste québécoise qui se dit « préoccupée par les transformations que subissent les paysages, [par le] spectacle de l'étalement urbain et [les] nombreuses disparitions qui l'accompagnent », elle s'intéresse « aux sentiments d'aliénation, de déracinement et de dislocation » $\left(\right.$ Hayeur $\left.^{6}\right)$ que créent ces phénomènes, tout en alimentant sa pratique aux problématiques environnementales et urbanistiques.

Ses premières séries photographiques « Paysages incertains7 » (1998-2002) et « Destinations ${ }^{8}$ » (20032004) présentent des panoramas paysagers, principalement de forêts et de lacs pour la première, un ensemble plus diversifié de routes, quartiers, bord de mer pour la seconde. L'impression d'harmonie « naturelle » qui se dégage à première vue de chacun des panoramas laisse cependant vite place au doute quant au réalisme du paysage représenté. En fait, chacun de ces panoramas est un subtil montage de 

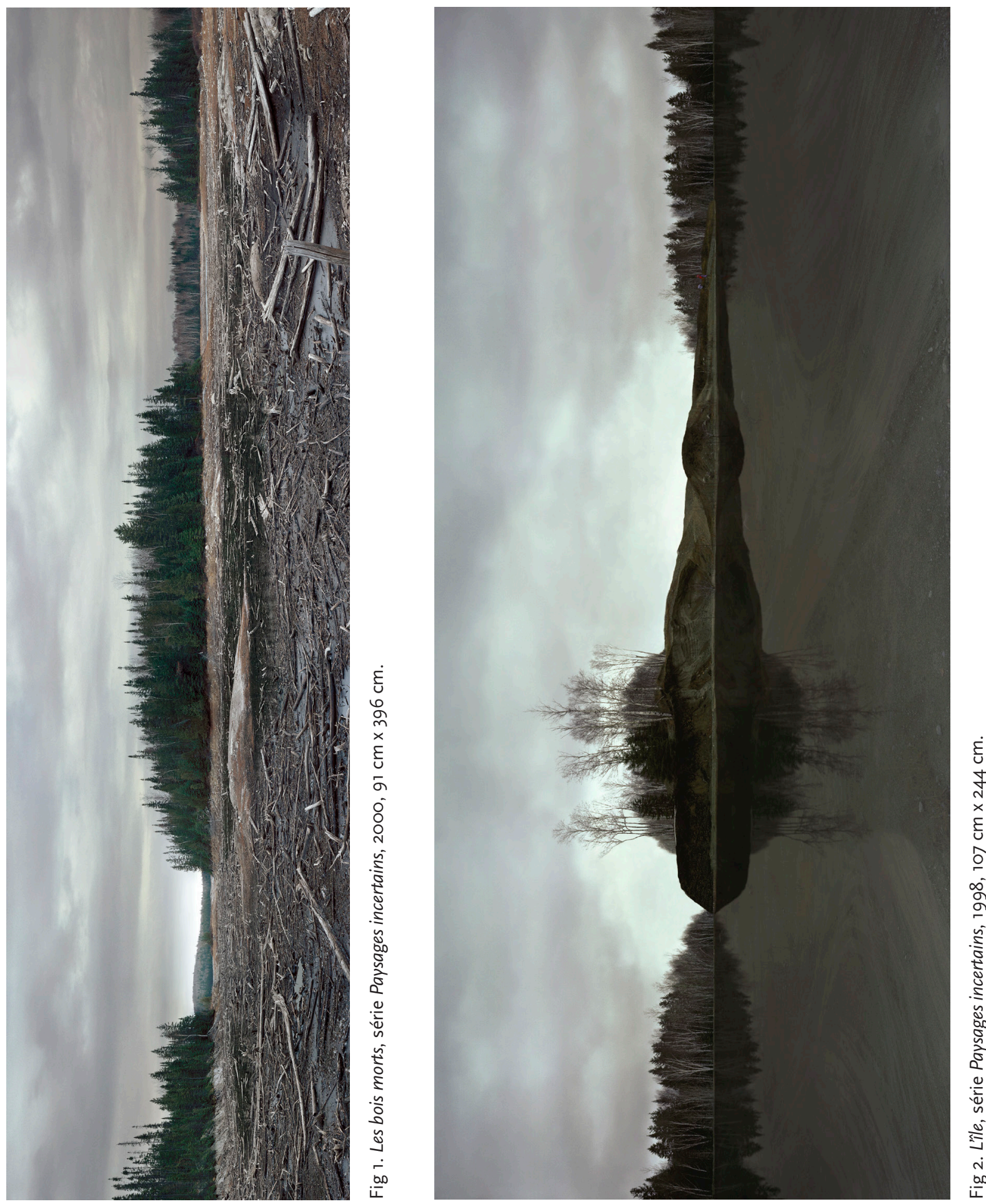

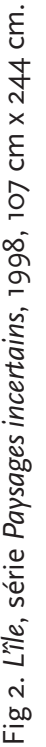



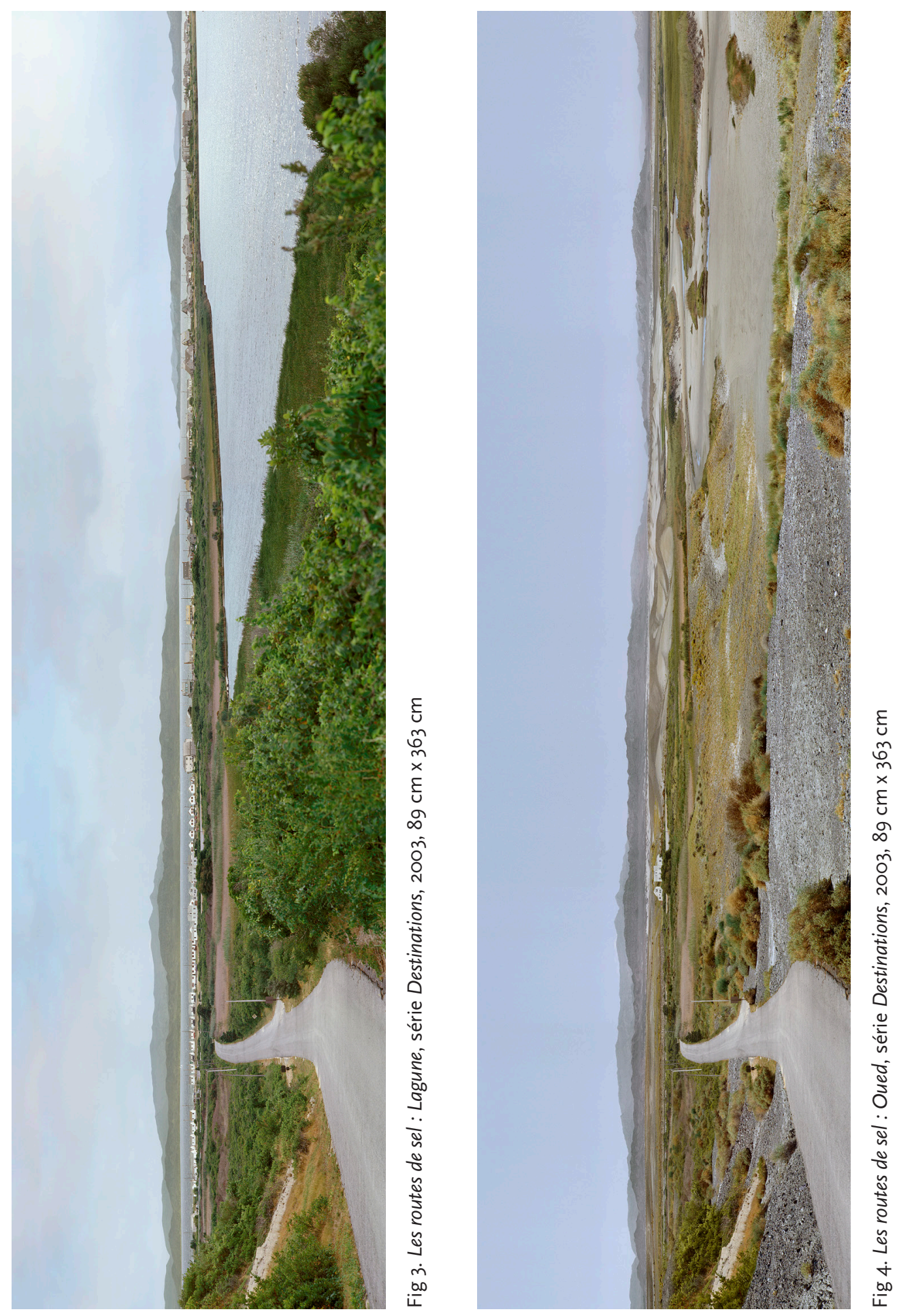
différents éléments réagencés qui produit une impression de réel, mais qui est en fait complètement artificiel. Au sujet des photographies de « Paysages incertains », Hayeur explique que «[l]es images de cette série ont été principalement élaborées à partir de photographies de réservoirs hydroélectriques et de rivières harnachées. » Si la photographie « Les bois morts9 » fait parfaitement illusion, d'autres laissent deviner que ce qu’elles donnent à voir est une nature réagencée.

«L'île $\mathrm{e}^{\mathrm{o}}$ », par exemple, est entourée d'une eau peu profonde qui laisse voir que son fond a été tourmenté par de gros engins mécaniques.

De façon encore plus ostensible, «Échappée ${ }^{\mathrm{II}}$ » montre l'ampleur et la profondeur de travaux en exposant les gravats et matériaux exhumés ou importés. La clôture grillagée en apparence très solide qui borde la tranchée sur une longue distance paraît flambant neuve et complètement déplacée en ce lieu dévasté. Cet élément entre également en contradiction totale avec le titre, dès lors ironique, de la photographie.

Comparons à présent le diptyque « Les routes de sel » dont les deux pans reproduisent la même route, perçue du même point de vue, mais transposée dans deux contextes différents évoqués par les titres.

« Lagune ${ }^{\mathrm{I} 2}$ » est construite selon des lignes fuyantes, légèrement diagonales, qui délimitent une grande moitié inférieure de la photographie en créant un contraste entre le vert vif qui occupe le centre et le bleugris de l'eau à droite. Ces perspectives créées par la route et le bord de l'eau viennent buter contre l'horizon de couleur très proche de l'eau et sur lequel se découpent de fins reliefs. Ce paysage photographique repose donc sur des contrastes d'éléments et de couleurs, agencés selon un réseau de perspectives nettes.

« Oued ${ }^{13}$ » tend beaucoup plus vers le monochrome, sa palette oscillant du brun pâle au jaune et au vert délavé. $\mathrm{Du}$ point de vue des éléments, nous sommes projetés dans un univers minéral à la végétation pauvre. La modeste étendue d'eau sur la droite reproduit de façon bien dérisoire la « lagune » de l'autre photographie. Cette relative uniformité des éléments et des couleurs rend la photographie plus difficile à lire. Certes, la route fournit un point de départ au regard, mais lorsqu'elle disparaît, celui-ci erre alors en quête de repères, perdus dans les dénivellations du milieu de l'image qui sont juste assez hautes pour obstruer la vue, mais pas assez pour créer un effet de surplomb.

Dans une série comme dans l'autre, l'harmonie et la quiétude qui se dégagent de chaque scène - au sens théâtral où la composition est une mise en scène paysagère - sont fabriquées de toute pièce car, à bien y regarder, l'œil finit par y trouver une faille, une intrusion suspecte. Comme l'écrit Hayeur, « [à] mi-chemin entre la zone et la carte postale, [ces images] font figures de maquettes ou de plans d'aménagement déroutants. Leurs étrangetés et leurs ambiguïtés deviennent une intrigue pour celui qui les regarde, produisant un sentiment partagé, hésitant entre le rejet et la fascination ${ }^{\mathrm{T} 4}$ ». La facticité de ces « paysages-spectacles » trahit notre attrait pour le sublime, les décors surfaits mais n'en laissant rien paraître. Plus encore, ils renseignent sur notre ambivalence à l'égard des paysages : on voudrait à la 


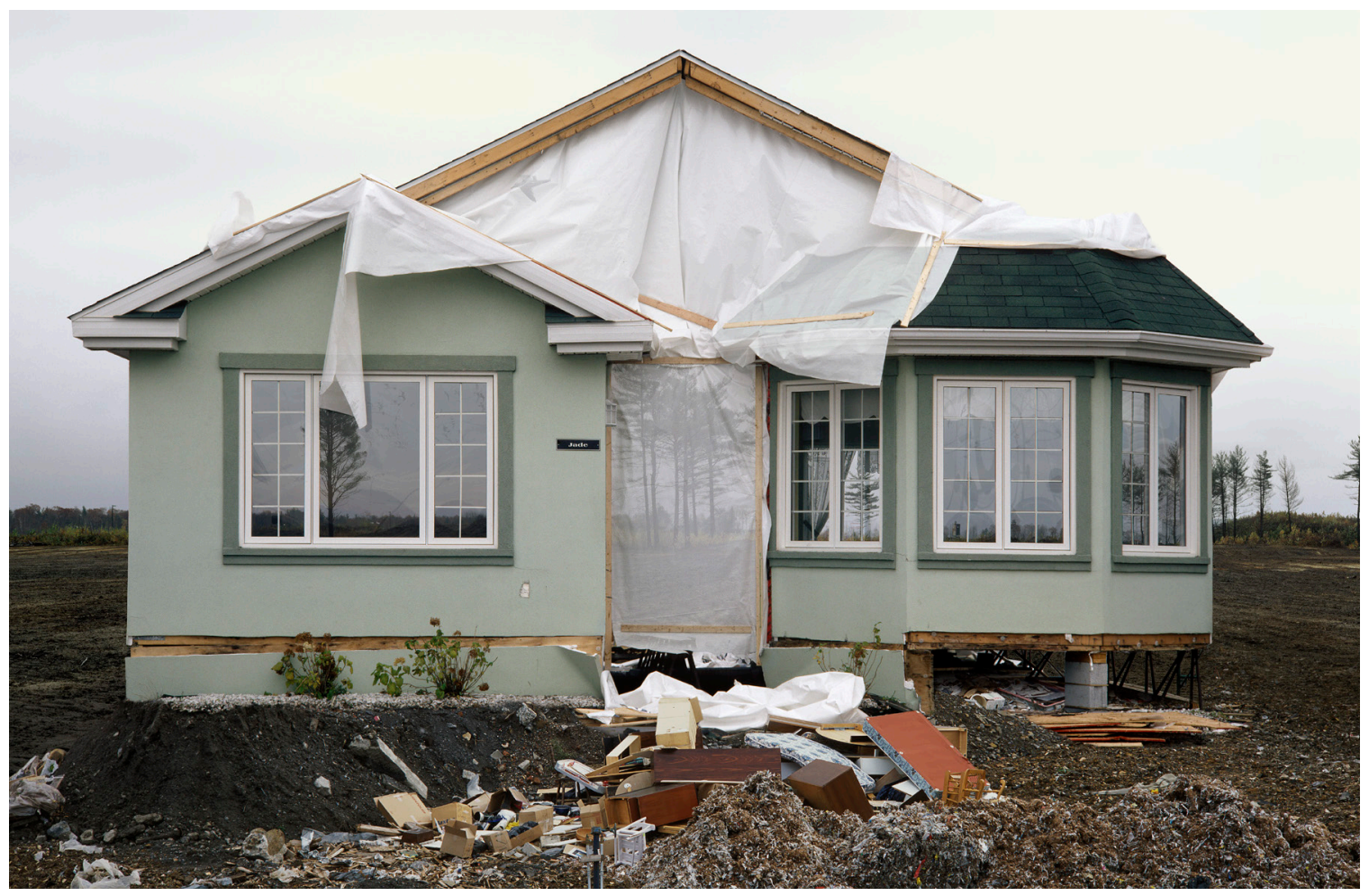

Fig 5. Jade, série Maisons modèles, 2004, $109 \mathrm{~cm} \times 157 \mathrm{~cm}$.

fois les maitriser - d'où la multiplication des « points de vue privilégiés à ne pas manquer » le long des routes panoramiques - et pouvoir s'y immerger - c'est-à-dire annuler toute distance au point de ne plus les percevoir comme paysages.

Les séries photographiques suivantes « Maisons modèles ${ }^{15}$ » (2004-2007) et « Nuits américaines ${ }^{16}$ » (2004-2008) explorent l'habitation nord-américaine. Comme la réalité des villes nord-américaines qui fait se côtoyer la plus criante démesure architecturale avec le délabrement le plus affligeant, ces séries mêlent bâtiments désaffectés, gratte-ciel miroitants, « McMansions ${ }^{17}$ », routes abandonnées rongées par le chiendent ou encore des maisons modèles de quartiers de banlieue résidentielle non encore achevées mais qui paraissent déjà fantomatiques sous leurs bâches de protection battant au vent.

« Jade ${ }^{18}$ », tirée de « Maisons modèles », est une maison qui manifestement a l'ambition de devenir coquette et confortable; mais pour l'instant, certaines de ses façades sont déjà achevées jusque dans les moindres détails, alors que des bâches font encore office de porte et de toit et que les rebuts de matériaux de construction jonche le sol inégal à l'avant de la maison. Hayeur a transposé cette maison dans ce qui semble être un désert ou un espace ravagé dont ne subsistent en arrière-plan que quelques arbres rachitiques. Par contre, les fenêtres et la porte de la maison reflète un paysage - ou la mémoire d'un paysage effacé? - composé d’arbres, mais qui est tout aussi déplacé que la maison. 
Au projet esthétique s'adjoint alors une critique sociale touchant à l'ampleur des disparités économiques, ainsi qu'une réflexion sur la diversification des banlieues après I980. Si l'apparition des premières banlieues dans les années I950-I960 frappait par l'anonymat et la standardisation de ces nouveaux quartiers, il est évident que le terme « banlieue » recouvre aujourd'hui une réalité qui s’est grandement complexifiée. En dépit de certaines affirmations, les banlieues ne sont plus parfaitement interchangeables. Ainsi que le souligne Laurent Lussier (2013),

[v]ue de la route, la banlieue n'a pas d'histoire. À l'inverse, lorsqu'on la traverse à pied, c'est son épaisseur historique qui s'impose au regard. La banlieue montréalaise est non seulement beaucoup plus complexe et diversifiée que l'image banale qu'on s'en fait, mais elle est aussi, à plusieurs égards, profondément québécoise et montréalaise. (21)

Son article sur « Le grand collage ${ }^{\mathrm{I} 9}$ » révèle ainsi les nuances de personnalité des banlieues qui s'échelonnent entre Oka et Saint-Hilaire afin que « la banlieue révèle la multiplicité de ses paysages » (2I). Les banlieues acquièrent des identités, des habitus et des modes de fonctionnement, tout en s'adressant à une population particulière. Or, ces identités et ces pratiques sont créées de toutes pièces, conçues puis vendues - bien souvent avant même que le projet résidentiel ne soit achevé. Elles fonctionnent comme des cadres dans lesquels vont se fondre les nouveaux résidents dont les interactions ne vont ensuite qu'influencer ce qui a été prédéterminé.

Enfin, les deux séries les plus récentes « Excavations ${ }^{20}$ » (2005-2008) et « Underworlds ${ }^{2 \mathrm{I}}$ » (2008-20I4) semblent s'éloigner des questions de la représentation et de la mise en spectacle pour traquer ce qui pourrait paraître comme l'origine des maux présentés dans les séries précédentes, mais en est plutôt un effet. La série « Excavations » donne à voir « les déserts que nous laissons derrière nous ${ }^{22}$ » lorsqu'un site est rasé ou creusé pour une construction. Aucune particularité locale n'est prise en compte, chaque nouveau site subit le même traitement (arasement, déblaiement de la terre arable, excavation) créant souvent pour de longues périodes de temps des espaces dénudés, laids - la terre apparaissant alors comme un corps lacéré. La série «Underworlds » sonde les conséquences de l'urbanisme contemporain sur les cours d'eau. Presque toutes les photographies de cette série montrent, au moins dans les deux tiers inférieurs, si ce n'est 90\% de leur espace, le désert aquatique malsain qui règne sous la surface de l'eau.

Surplombé dans la partie supérieure de la photographie par des maisons, des édifices ou différents arbres pris en contre-plongée, cet inframonde frappe par son opacité et sa saleté. La ligne de la surface semble délimiter deux mondes aveugles l'un à l'autre qui sont en fait intimement liés et interdépendants. 


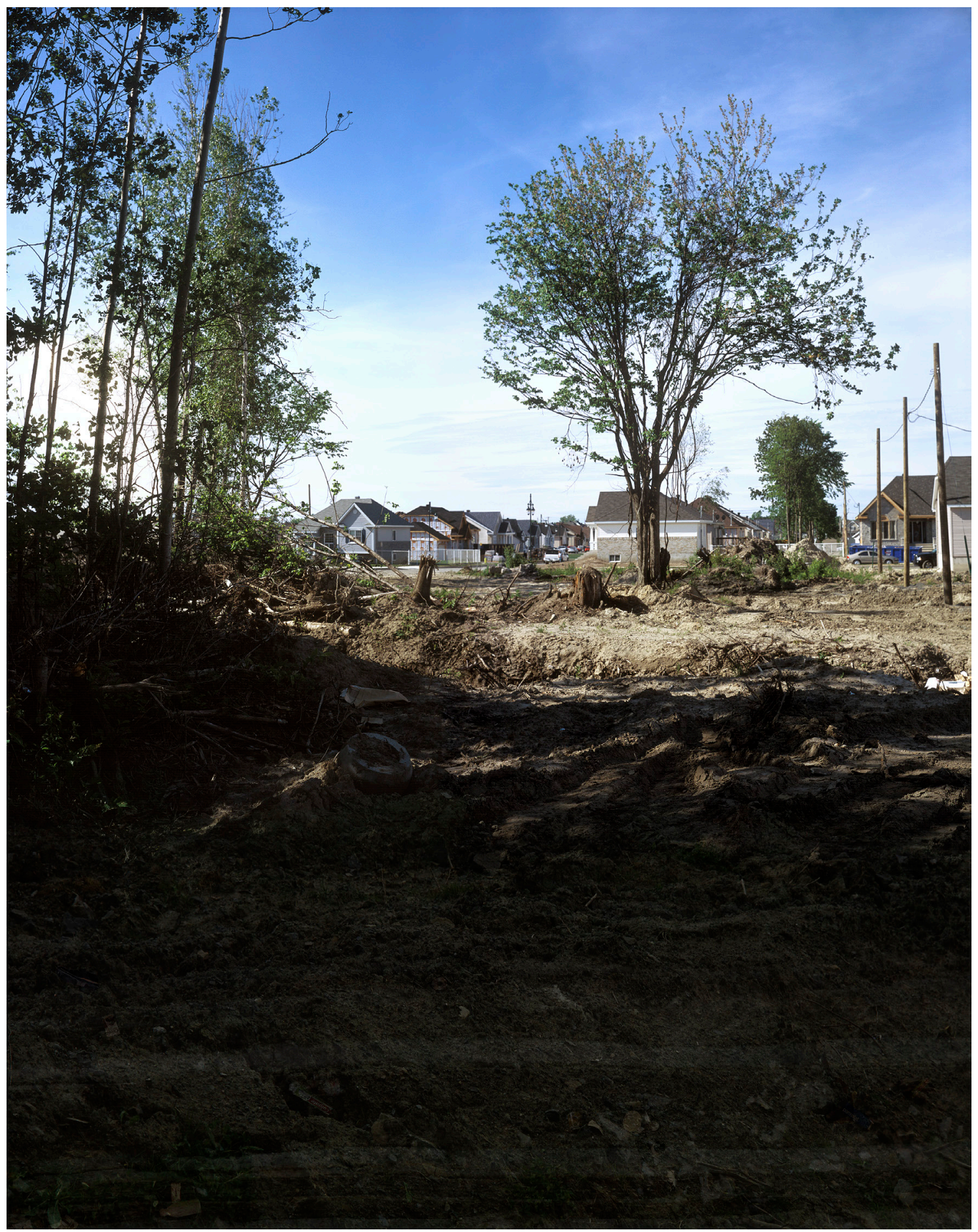

Fig. 6. Blindsight, série Excavations, 2005, $267 \mathrm{~cm} \times 152 \mathrm{~cm}$. 


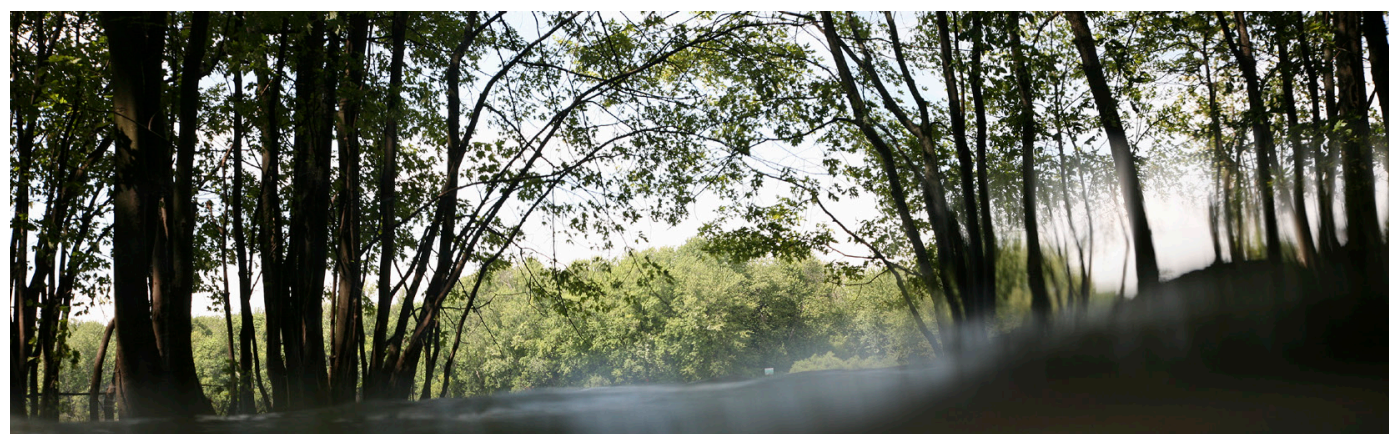

Fig 7. Blackwater, série Underworlds, 2010, 153 cm x $111 \mathrm{~cm}$. 


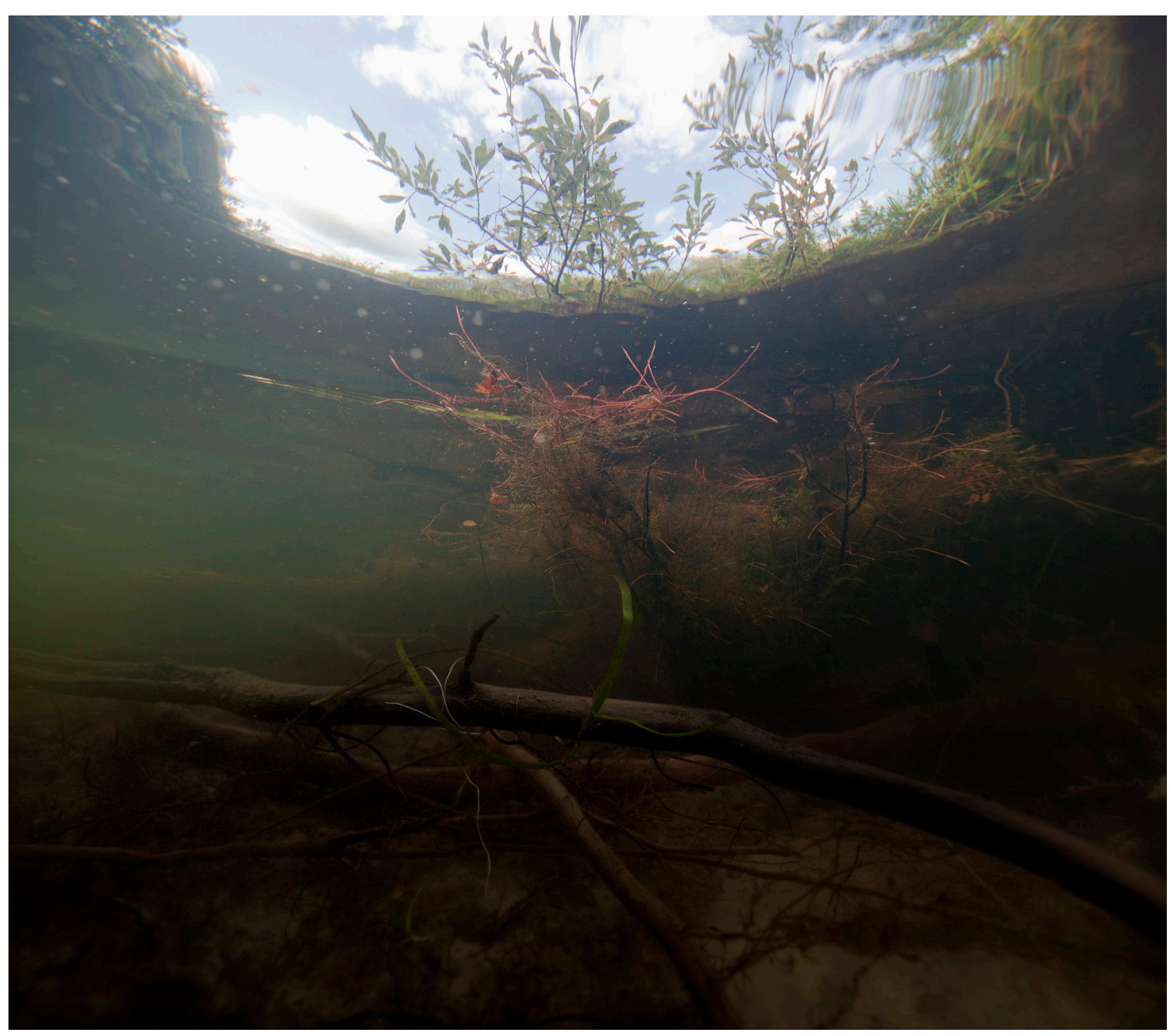

Fig. 8. Lac Champlain 01, série Underworlds, 2013, $122 \mathrm{~cm} \times 107 \mathrm{~cm}$.

Depuis 2009, presque une dizaine de vidéos ont été élaborées parallèlement à ces séries photographiques. Hayeur explore ici dans un autre langage certains sujets qu'elle avait abordés par la photographie précédemment. Ainsi, «Losing Ground » (2009), « Private Views » (20I0) et « Déraciné » (20I2) interrogent à leur tour les disparités sociales et leur visibilité dans le paysage contemporain. Par des effets de surimpressions et de fondus enchaînés, l'artiste passe de maisons placardées tombant en ruine à des maisons tout juste sorties de terre, du fond d'une piscine dans une résidence de luxe au fond glauque et inquiétant d'un lit de rivière. Dans ces œuvres, le langage est totalement désert; il n'y a aucun texte à lire ni aucun propos sonore. On entend seulement une musique intermittente, le vent dans les branches, dans des bâches ou encore le bruit de la circulation. Et pourtant, le discours qui sous-tend ces vidéos est éloquent. Isabelle Hayeur y donne à voir ce que chacun est habitué à voir, mais à travers un regard qu'on 


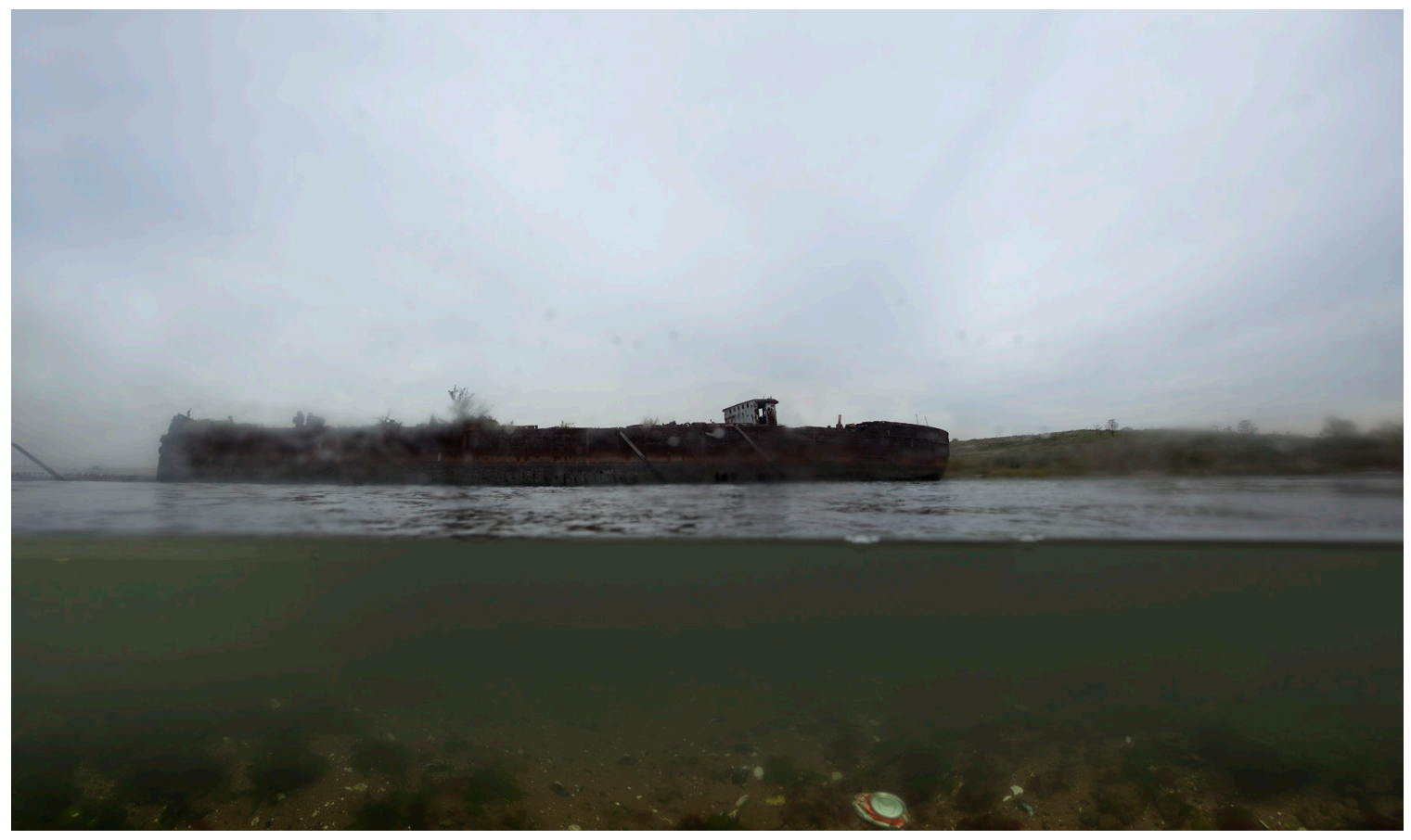

Fig 9. Death in Absentia 01, série Underworlds, $2011,108 \mathrm{~cm} \times 183 \mathrm{~cm}$.

qualifierait volontiers de clinique, à la fois distant et froid en cela qu'il se présente comme radicalement extérieur à ce qu'il décrit.

« Losing Ground ${ }^{23}$ » commence par l'image d'un mince rideau d'arbres qui frissonnent dans le vent. Un zoom arrière fait apparaître un cadre de fenêtre par lequel était vue cette première image. Il s'agit de la fenêtre d'une vieille maison de pierre à l'abandon, envahie par la végétation et les repousses. Un travelling partant de cette bâtisse donne à voir un nouveau projet résidentiel construit tout près, qui était quelque peu discernable à travers les arbres. Ce balayage assez rapide se stabilise sur un de ces McMansions mentionnés plus tôt, non encore achevé et couvert de bâches battant au vent. Une musique aux accents inquiétants et parcourue de disruptions électroniques s'amplifie alors, tandis qu'un second grand travelling circulaire débute, comme si d'une auto en mouvement l'on voyait défiler tous les environs de ce nouveau projet qui aligne les rangées de gros bâtiments. À l'arrière d'un centre commercial, la caméra se fixe sur des traces de sang maquillées sur la route. Les voitures roulent dessus à vive allure, alors que des effets spéciaux les font passer au ralenti et les font paraître fantomatiques. Cet épisode tout comme celui de la vieille maison abandonnée - à laquelle revient la caméra à la fin - pose la question de l'effacement mémoriel, qu’il s'agisse d'une mémoire ancestrale, comme dans le cas de la maison traditionnelle et qui sera sans doute bientôt condamnée, ou d'une mémoire à court terme, presque instantanée. Les traces de sang sur la route ont quelques heures, quelques jours tout au plus, mais les voitures contribuent à leur effacement sans 
y prêter la moindre attention. Sitôt clos, l'incident a déjà basculé dans l'oubli. Et de fait, la caméra passe ensuite à une vue en plongée d'un bain à remous privé autour duquel rient des femmes. Par un effet de fondu enchaîné, la caméra donne l'impression de plonger dans ce bain bouillonnant pour en révéler les bas-fonds. On voit alors le fond d'un lac ou d'une rivière avec poissons et algues brunes et filandreuses, tout un inframonde peu rassurant et glauque, impression renforcée par le retour de la musique en arrièrefond. Lorsque la caméra remonte à la surface, elle se trouve de nouveau devant la vieille maison, sur fond de musique dissonante. L'image finale consiste en un gros plan sur des débris de la maison tombé au sol, indice ultime de l'effacement.

Fonctionnant comme un diptyque, « Private View ${ }^{24}$ » est la vidéo qui offre le contraste le plus saisissant entre extrême pauvreté et extrême richesse. Le titre, sur fond blanc, apparaît sur fond de bruit de circulation. La caméra opère un mouvement descendant, comme si elle descendait du ciel pour se fixer sur une immense et vieille demeure, complètement abandonnée à présent. Décrépite, ornée de graffitis, les ouvertures de cette habitation sont placardées. La vidéo donne ainsi à voir de nombreuses maisons dont le passé et les dimensions varient, mais qui toutes sont désertées. Leur enchaînement de plus en plus rapide suggère leur grand nombre, comme si la présence de ces bâtiments à la dérive en plein paysage urbain n'importait pas, comme si cela n'avait aucune signification. La caméra s'arrête enfin sur l'une de ces maisons et opère un zoom sur l'une des fenêtres, donnant l'impression d'y entrer. Les plans suivants donnent à voir un intérieur complètement délabré, à l'image de la façade extérieure. Lors d'un fondu, une musique d'ambiance apaisante commence et le fondu s'achève alors sur un tout autre intérieur, à la fois récent et luxueux. Toute la seconde partie de la vidéo montre alors de prétentieuses demeures semblables à des châteaux de pacotille. La vidéo prend fin sur l'une d'elles que l'on voit entre les barreaux de sa grille de fer forgé fermée. Par un subterfuge, la grille devient portail noir plein, annonçant le fondu au noir final.

« Déraciné25 » revient sur les grands projets résidentiels, en posant un regard insistant sur les coupes de bois nécessaires pour dégager le terrain, l'encombrement de poteaux électriques que tout nouveau quartier suppose, la pollution des cours d'eau avoisinants, ainsi que les gros travaux au bulldozer qui marquent la terre. Les images montrant les traces des gros engins de construction dans la terre la font paraître comme un corps ravagé, couvert de plaies ouvertes, sales et incicatrisables. « Toutes ces plaies pour si peu », semble suggérer la vidéo lorsqu’ensuite elle montre l'érection de rangées de maisons standardisées flambant neuves, et ce, à perte de vue. Un autre point de vue montre ensuite un immense stationnement désert, corollaire perçu comme indispensable au mode de vie impliqué par ces maisons, sans oublier les bretelles d'autoroute et autres voies d'accès. Hayeur place ici sa caméra légèrement en contrebas, comme si elle adoptait la hauteur de vue d'un petit animal, raton laveur ou lapin. Le bruit des voitures est à son maximum et la vue imprenable sur les déchets qui jonchent le bas-côté. Elle filme ensuite à ras de terre quelques rares pousses végétales qui, par effet de fondu, deviennent des cheminées d'usines fumantes, le tout sur fond de musique électronique. 
L'indéniable qualité esthétique de ces quelques œuvres d'Isabelle Hayeur donne lieu à un regard pénétrant et bien particulier. Les plans, les effets visuels et sonores, bref tout le montage dans son ensemble concourent à créer une œuvre extrêmement construite qui agence ses effets pour renforcer son discours critique et les interrogations qu'elle pose. Hayeur semble en effet avoir entendu et fait sien l'appel que lançait Simon Harel (2007) dans Espaces en perdition : « Je plaide pour l'investigation de tactiques marginales qui posent la question cruciale de l'usage des territoires que nous habitons » (58). « Marginales », « les tactiques » et stratégies de Hayeur le sont à bien des égards; elles mettent évidemment en question «l'usage des territoires que nous habitons », mais elles vont plus loin également que cet usage en y adjoignant la dimension paysagère, c'est-à-dire esthétique. Il est difficile ici de déterminer à quel point pratique et esthétique sont exclusives l'une de l'autre ou encore tellement intriquées qu'elles en deviennent consubstantielles. Décider que pratique et esthétique s'excluent mutuellement revient à dire que tout dispositif donnant priorité à la pratique laissera à désirer du côté de l'esthétique - et sous-entend la réciproque. Mais peut-être est-ce davantage une imbrication des deux qu'il faut percevoir : tout dispositif sous-entend une logique circulaire, ainsi que le laissent comprendre les vidéos. Un projet conçu avant tout en fonction de rentabilité (à la construction) et de commodité (pour les usagers et habitants) suppose ou tolère un moins grand raffinement, moins de personnalisation dont on s'accommode pour les avantages cités. Du moment où l'esthétique passe au second plan pour la construction des habitations, pourquoi n'en serait-il pas alors de même pour les commerces, les routes et les abords? Tout tend alors vers la logique du moindre effort. Au contraire, dès que l'un de ces aspects est soigné, chaque élément du paysage dans lequel il s'intègre doit alors témoigner du même souci, de la même qualité. Nancy Huston (I995) exprime en des termes percutants un constat similaire :

Comment se fait-il que, dans le Nouveau Monde [...] nous soyons si fermés à cet aspect esthétique de la vie ? que l'on ne se soucie plus de transmettre aux générations futures de la beauté ? que l'on estime normal de mettre devant les yeux de nos enfants un enchaînement chaotique de restaurants fast-food, de stations-service, de bâtisses disgracieuses et de centres commerciaux ? Comment faisons-nous pour croire que cette hideur ne déteindra pas sur leur âme ? (14-15)

Ce qui se joue dans ces lignes est l'une des questions sous-jacentes à l'œuvre de Hayeur : comment les paysages participent-ils à la définition des goûts esthétiques du sujet. S'il est vrai, ainsi que nous l'avons souligné en début de réflexion, que le sujet est celui qui découpe et donne forme au paysage à partir d'une étendue de pays, il est tout aussi vrai de dire que les paysages façonnent en retour notre regard et nos valeurs. L'interaction entre sujet et paysage est constante. Les œuvres de Hayeur sont avares de présence humaine. On ne remarque que quelques figurants sur certaines vidéos, l'essentiel de l'image étant consacré aux arbres, au bâti, à un ciel vide écrasant ou à un monde aquatique vaguement inquiétant, ou encore au vent, toile de fond sonore de plusieurs vidéos, qui fait tantôt frémir branches et feuilles, tantôt battre d'immenses bâches, la part dévolue tantôt au ciel et tantôt au contraire au monde subaquatique. Pourtant, la préséance revient toujours pourtant finalement au sujet situé hors champ, placé derrière l'objectif ou la caméra. Cependant deux raisons conduisent à affirmer cette primauté du sujet sur le paysage dans son 
œuvre : d'une part, les paysages qu'elle construit soulignent généralement l'influence néfaste des activités humaines sur le paysage; d'autre part, son regard et son travail de composition (notamment à travers l'altération de photographies ou des jeux de surimpressions dans les vidéos) déterminent notre perception des paysages qu'elle élabore.

En est-il toujours de même dans les démarches artistiques contemporaines? Il semblerait bien que cela soit une tendance majeure tant dans de récentes expositions d'arts visuels que dans la littérature, objet du dernier temps de notre réflexion. On pourrait dénombrer plusieurs romans québécois ${ }^{26}$ parus au cours des dernières années dans lesquels une attention particulière est portée aux paysages; mais c'est la poésie qui retiendra ici notre attention.

\title{
Paysages postindustriels en poésie
}

Les représentations paysagères en poésie québécoise contemporaine accordent beaucoup d'importance à la subjectivité. Comment d'ailleurs pourrait-il en être autrement pour un genre littéraire qui porte à son plus haut point l'exigence d'exprimer une subjectivité absolument singulière? Dans la plupart des exemples que nous allons examiner, l'influence de l'activité humaine, ses aménagements paysagers et ses choix en matière d'urbanisme sont déterminants. Le poème met en mots le résultat, c'est-à-dire le nouveau paysage qui en naît coloré de toute la gamme d'émotions qu'il suscite chez le sujet.

Dans Lignes aériennes (2002) de Pierre Nepveu, chaque section joue un rôle bien particulier dans ce recueil consacré à la construction et à la déchéance de l'aéroport de Mirabel. La première («Approches ») et la dernière (« Retour ») campent le Je en promeneur contemporain du lecteur, citadin lancé dans une quête dans les environs de Mirabel. Sa quête, d'ordre ontologique, mêle passé individuel et collectif, sentiment de perte et sens :

\author{
J'ai cru qu'un recommencement \\ m'allègerait de tout besoin \\ et que s'écrouleraient les voies du passé \\ $[\ldots]$ \\ je voudrais que des paroles \\ ardentes, fortement proférées \\ accompagnent mes progrès dans ce monde \\ mais je sens mes mains agripper le vent, \\ il y a comme un creux par-devant \\ une éclaircie lunaire aux contours vagues [...] (16)
}


À travers un contre-rejet exemplaire, le sujet exprime le souhait de «paroles/ardentes, fortement proférées ». Il est donc en quête d'un récit vivant et signifiant; mais on pressent déjà que « le vent », le « creux pardevant » et l'« éclaircie lunaire aux contours vagues », toutes expressions qui insistent sur le vide, auront raison de cette quête qui ne débouchera que sur une aporie, un sentiment de désarroi ou de deuil encore plus marqué. La section centrale, « Mirabel », constitue une vaste analepse pour laquelle le poète imagine plusieurs personnages, tels que l'arpenteur ou la femme de ménage, et auxquels il délègue la parole. Le « Cahier de l'arpenteur, été I969 » révèle le paysage que va faire advenir ce personnage démiurgique :

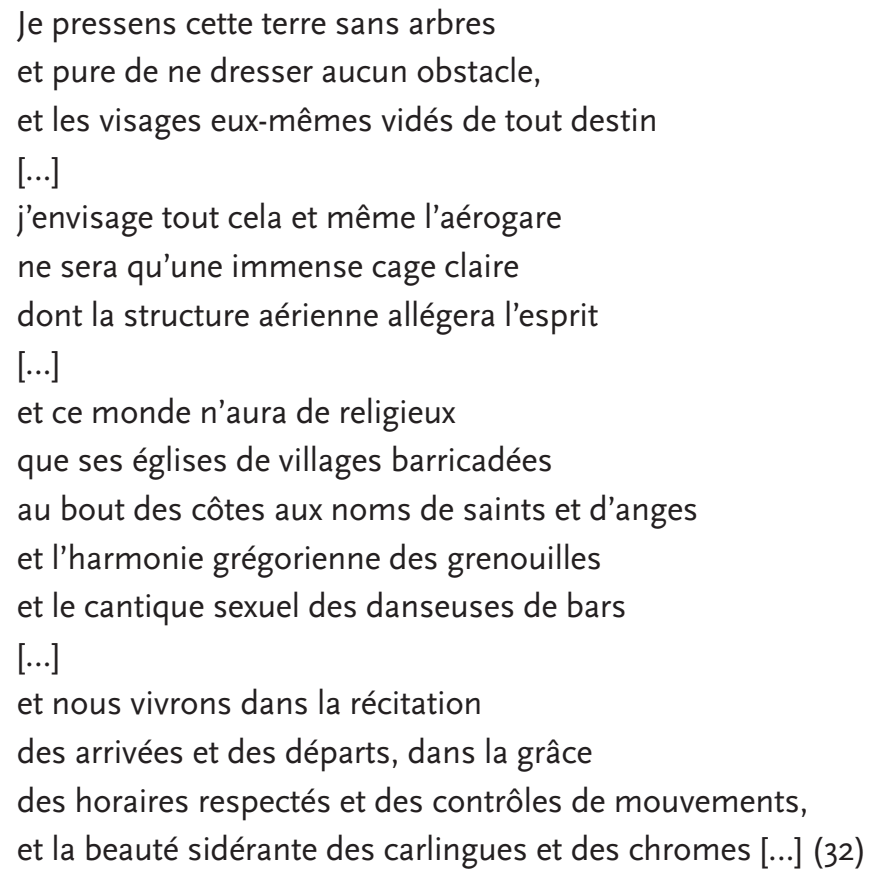

Ce paysage est aussi bien plus tant sa description révèle également ce qui demeure invisible, soit l'intériorité et la spiritualité des habitants. Le Je lit ici «cette terre » tout comme il lit les visages qui l'habitaient au sens plein jusqu'alors. Il faut remarquer comment l'adjectif « pure », détaché du nom qu'il qualifie, crée un malaise. Il n'est pas synonyme ici de pureté originelle, mais bien de l'adjectif « vidés » introduit au vers suivant. Ce que le Je pressent, c'est ce passage d'un paysage peuplé et signifiant à un néant esthétique et existentiel. L'aérogare, qui devrait être perçue comme un lieu de départ, de possibilité d'envol, n'est, par tournure restrictive, « qu'une immense cage claire », autrement dit ce qui au contraire retient, emprisonne, plutôt que de libérer. Ce poème exemplifie ensuite le procédé de détournement du religieux qui est à l'œuvre dans tout le recueil en associant de façon grotesque le vocabulaire religieux à des éléments relevant du plus complet prosaïsme ou d’une sexualité dépravée : «l'harmonie grégorienne des grenouilles » est mise en parallèle avec « le cantique sexuel des danseuses de bar », tandis que plus bas, par le même phénomène de contre-rejet que celui déjà vu précédemment, Nepveu place le terme religieux en 
fin de vers avant de détourner son sens au vers suivant : «la récitation / des arrivées et des départs », «la grâce / des horaires respectés et des contrôles de mouvements ». Le dernier vers cité prolonge cet effet : la seul beauté digne de ce nom qui subsistera ne sera pas d'ordre spirituel mais extrêmement matériel, celle « des carlingues et des chromes ». Plus que d'être vécue de façon à la fois collective et intime, la foi se trouve donc réduite à de simples inscriptions extérieures (les noms de côtes) et à des détournements parodiques avilissants. Si de nombreux poèmes sont voués à la description des environs, à donner à voir Mirabel à travers des vues panoramiques ou au contraire des petites vignettes très ciblées, un nombre tout aussi important d'autres montrent l'envers du décor, c'est-à-dire les fermes abandonnées, les villages « travesti[s] en banlieue » (Nepveu 2002, 9I) et les détresses existentielles que ce projet a provoqué :

Le dernier homme se penchait parfois

à sa fenêtre en guettant les présages

du printemps ou de la fin du monde.

Les deux arrivèrent de concert,

dans un cliquetis de chaînes fermant

le rang et les bouffées d'un incendie

qui ravagea la grange et des carrés

de foin encore jaune et laissa

l'homme pantois au bord d'un fossé [...] (42)

Ce fossé rappelle étrangement le « creux par-devant » que pressentait le sujet au début du recueil. Ce poème du bord de l'abîme flirte avec l'eschatologie; la fin d'un monde ici indique la fin d'un paysage et des habitus qui le façonnaient. Le « cliquetis de chaînes fermant / le rang » rappelle le sème de l'emprisonnement introduit par la « cage claire » (32) et « les bouffées d'un incendie » (42) étaient préfigurées par l'« éclaircie lunaire aux contours vagues » (I6). On voit donc plusieurs motifs ressurgir au fil du recueil et se moduler. Tous pointent l'effacement du paysage traditionnel au profit d'un paysage reconfiguré, méconnaissable et qui travestit ses valeurs. La fin du recueil renoue avec l'idée d'une quête qui demeure sans réponse : « Les lignes aériennes sont peu lisibles / dans le ciel » (Nepveu 2002, I06). La polysémie du titre du recueil joue sur les lignes des compagnies aériennes, les traces impalpables que les avions laissent dans le ciel et les vers éthérés du poème. L'effacement, la perte de sens et la difficulté à déchiffrer sont au cœur de ces trois significations. De fait, le Je admet avoir laissé un peu de lui-même dans cette entreprise :

Quelque chose en moi, d'être allé plus loin

que moi-même, n'a pas voulu revenir

$[\ldots]$

je réfléchis sans mots, je tremble sans même

l'apparence d'un corps, la densité d'une peau

je m'avance fantôme dans une rue

qui ressemble à la mienne et je frappe

à la porte d'une hôtesse généreuse 
qui a tous les traits de la femme

qui faisait ma vie, elle ignore

que je ne suis pas revenu [...] (108)

Si le sujet tend à s'effacer, voire à se musser derrière des personnages dans Lignes aériennes, il reprend pourtant à la fin l'initiative de la parole, mais ce n'est que pour constater la perte de sa subjectivité. Il se décrit comme «fantôme » « sans même / l'apparence d'un corps », un être qui n'est « pas revenu » intact de sa quête qui l'a laissé « sans mots » et «trembl[ant] ». Son état d'esprit, mélange d'infinie tristesse, de vacuité et de colère, donne le ton à l'ensemble. L'aéroport, lieu iconique du village global aux distances réduites par la technique et la technologie et ouvert sur le monde, n'est pourtant ici en rien un lieu de rencontre. Monde technique clos sur lui-même, il est un espace exclusif, une greffe forcée qui n’a pas pris dans la région environnante.

Dans Reculez falaise (2007), Louis-Jean Thibault élit un « site » de la ville de Québec pour chaque poème, du « Château Frontenac » à une « Rue anonyme », des « Plaines d'Abraham » au « Cimetière Saint-Charles ». Le poème liminaire pose un enjeu qui relève à la fois de l'intimité du sujet et de son intérêt pour la ville :

Que cherche-t-il encore ici (quarante-septième parallèle Nord, soixante et onzième Ouest), il ne saurait clairement le dire.

Les lointains se sont rapprochés, mais les multiples lieux que déroule à ses pieds le paysage où il est né lui apparaissent davantage mystérieux avec leur infinité de détails que l'œil attire vers lui comme un aimant affolé.

[...] il s'enfonce, espérant trouver - belle et grande utopie le centre de gravité de la ville ou de lui-même [...] (11)

Ce poème annonce un sujet au regard renouvelé, scrutateur, attentif aux moindres détails du paysage urbain et des sensations qu'il suscite. Dans la chronologie du poème, tout indique que les lieux dont il va être question sont extrêmement familiers au sujet (« encore », «le paysage où il est né »), mais l'enjeu va précisément consister à dépasser cet « affol[ement] » superficiel de l'œil pour « s'enfonce[r] » dans l'épaisseur de la matière, pour effectuer une stratigraphie mémorielle de ces lieux. Cependant, ces lieux étant le théâtre sur lequel se joue sa vie ordinaire, le sujet suppose qu'ils peuvent renfermer « le centre de gravité de la ville ou de lui-même ». C'est à cet essai de stratigraphie que convie le poème « Quartier SaintRoch » qui décrit les transformations de ce quartier réaménagé devenu une sorte de palimpseste tant le récent vernis dissimule à grand-peine la véritable âme du lieu :

$[\ldots]$

En dix ans, le quartier a rééquilibré ses toitures et savonné 
ses artères, mais la peau perce encore sous le maquillage.

Le noir des regards se confond au sable cristallisé des vitrines :

la faim, le luxe ostentatoire, la rage, l'hystérie passagère.

Pour se protéger contre les maux, il ne semble y avoir aux alentours

que les vitraux des arbres adossés à la falaise, où tombe la lumière

du jour, qui hésite, depuis une heure, entre la sécheresse et l'orage. (67, nous

soulignons)

La métaphore corporelle, bien répandue pour évoquer le corps urbain, est ici filée avec habileté pour mettre à jour les cache-misère (« savonné », « artères », « peau », « maquillage). Le premier terme renvoie à un autre champ sémantique associé traditionnellement à la ville, l'hygiénisme, tandis que le dernier constitue un lointain écho à Baudelaire, poète de la modernité citadine. L’intermittence de la lumière à la fin répond à la discontinuité visuelle créée par ce paysage incertain, qui vacille entre chaos intrinsèque et nouvel ordre imparfait, tel que l'expose l'accumulation du vers « la faim, le luxe ostentatoire, la rage, l'hystérie passagère ». Mais cette lumière hésitante répond également aux incertitudes du sujet : «J'arrive mal à pousser un pied devant l'autre et le monde m’apparaît / comme un défilé sans fin de choses disparates, un bric-à-brac / où l'on épuiserait en vain ses forces à faire régner l'ordre » ( 67 , nous soulignons). Dans ces vers, le Je renonce à la tentation d'un regard lisse et consent à la bigarrure du lieu. Ce poème décrit le résultat assez peu convaincant d'une tentative de gentrification qui ne trompe personne. Au cours de ce processus, le lieu a manifestement perdu son unité, aussi grise était-elle dans le passé, pour produire à présent une insurmontable impression de « bric-à-brac », de bricolage inachevé. Le poème tire moins sa source d'une critique de ce processus ou de son résultat que du sentiment d'incertitude, d'hésitation, d’intermittence que crée maintenant ce lieu, et plus généralement, la mémoire stratifiée de la ville.

Dans Le paradis des apparences (2004) de Robert Melançon, le sujet est, comme chez Thibault, un infatigable promeneur citadin à l'œil aiguisé. Ces deux points communs - la déambulation en ville et l'observation - sont à nuancer par deux différences qui séparent leurs démarches : la ville, chez Melançon, est toujours Montréal, même si elle n'est jamais nommée dans ce recueil, et ne l'est que rarement dans les précédents. Alors que le sujet chez Thibault s'attache à faire ressortir «l'âme » de Québec en mettant en mots différentes facettes de la ville, en les colorant parfois de son propre vécu - notamment dans la section «Intime »-, Melançon a pour projet de produire un « essai de poèmes réalistes », si l'on s'en tient au soustitre de son recueil. « Je m’en tiens au paradis des apparences » (36), écrit-il, se donnant pour contrainte cette exigence de se restreindre au visible seulement : « on ne notera pas qu'il vente : on ne voit pas / Le vent, et on ne dira que ce qu'on voit » (130). De fait, le poète tient son pari en se tenant à distance de toute métaphore filée qui pourrait l'éloigner du réel. Seule la médiation picturale, très présente, trouve grâce en cela que la peinture éduque l'œil et influence ses perceptions. Plusieurs poèmes font donc allusion à des peintres très différents : la lumière « avère / La peinture de Seurat : poussière / Rose, bleutée, violacée » (23), tandis qu’à la page suivante, « Un gros cumulus flotte sur une mer bleue; / On dirait un ciel de 
Poussin » (24). De fait chez Melançon, pour aussi réel, concret et prosaïque que ce soit le paysage, il est toujours également tenu à distance et intellectualisé par le sujet, que ce soit par ces références à la peinture paysagère ou par des formulations paradoxales qui montrent du doigt les clichés à ne pas reproduire mais qui, par le fait même, les inscrivent dans une sorte de second degré dans le poème. Ces deux poèmes concernant l'automne permettent de voir ce mécanisme à l'œuvre :

L'automne s'y étage en un spectacle qu'on admire,

Prévisiblement, comme chaque année. On a raison

Quoiqu'il en soit, de le faire : tous ces jaunes, ces rouges,

Ces roses coupés par le vert des sapins, on a raison

De venir si loin s'en émerveiller. (10, nous soulignons)

L'adverbe « prévisiblement » sert manifestement ici à marquer une distanciation du sujet face au cliché afin que son adhésion au paysage ne soit pas complètement béate, tout comme dans cet autre poème le passage dégradant de la métaphore du « cliché sépia » à celle du « sac / De lieux communs » :

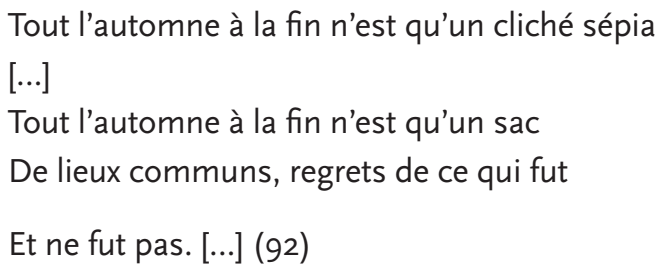

À cet égard, les paysages de Melançon sont aussi réels qu'intellectuels et culturels, ou pour le dire autrement, le paysage réel est toujours perçu par le filtre subjectif de paysages déjà connus, que ce soit dans le monde réel ou par la peinture. Dans le poème suivant, le printemps existe autant «sous les pas » qu'à l'intérieur du « crâne », et l'on marche autant « dans la rue » que « dans l'idée » qu'on se fait de cette rue. L'adjectif « verte», court et commun, condense toutes les platitudes qui pourraient être égrenées au sujet du printemps; mais son extrême retardement à la fin de la première strophe et son détachement du reste du vers le placent en position forte. La couleur verte fonctionne comme un point de jonction entre le réel (« L'étendue » « dans le parc » que le sujet a sous les yeux) et l'impression chromatique gardée à l'esprit des printemps précédents. L'adjectif forme donc le point d'orgue sur lequel se rejoigne la perception visuelle du moment et la représentation idéelle forgée à travers des expériences antérieures :

L'étendue que déploie le printemps,

S'étale dans le parc et dans la mémoire,

Sous les pas et sous le crâne - verte. 
On marche dans la rue que le soleil Repeuple de promeneurs et dans l'idée Qu'on se forme d'une rue en avril.

On passe à tout instant de la chambre Qu'on édifie de souvenirs vrais ou faux, À l'entassement des faits minuscules

Dont se fabrique chaque instant [...] (62)

L'expérience paysagère ainsi que son rendu poétique s'effectuent dans un va-et-vient constant entre les perceptions sensorielles au présent et la mémoire de perceptions plus anciennes.

Les poèmes du Carnet d'un improbable été (2013) de Claude Paradis font leur miel de la lenteur de la maison et du jardin le temps d'un été. Mais il est quelques poèmes qui s'en écartent un peu et laisse deviner les environs :

$$
\begin{aligned}
& \text { Je demeure dans une ville qui n'était } \\
& \text { jadis qu'un village autour duquel } \\
& \text { gravitaient quelques fermes. La banlieue } \\
& \text { a transformé le paysage et bouleversé } \\
& \text { le rythme de la vie. [...] } \\
& \text { [...] Tout est en général } \\
& \text { conforme au rêve américain. Le poinçon } \\
& \text { du marcheur ne s'enfonce plus librement } \\
& \text { sur le bitume noir des rues; personne } \\
& \text { ne nous regarde tenir l'ivresse des livres } \\
& \text { qui gardent ouverte la voie des rêves. (50) }
\end{aligned}
$$

De façon plus marquée que chez les autres auteurs, le sujet effleure le paysage pour évoquer davantage les habitus que celui-ci porte - ou ne porte plus. Le sentiment de regret, de perte entre le passé et le présent est palpable. Lorsque le monde visible est altéré demeurent cependant possibles quelques gestes ou habitudes qui subsistent, résistent à la façon de traces, témoignant d'un passé autre. Il ne s'agit pas de redonner vie à des souvenirs, mais bien seulement de les évoquer, de les faire surgir comme des témoignages d'une aire / ère révolue :

$$
\begin{aligned}
& \text { [...] Quelques villageois } \\
& \text { conservent d'anciennes habitudes : ici, } \\
& \text { un homme s'affaire à réparer la clôture } \\
& \text { d'un potager sur un rare terrain encore } \\
& \text { sans piscine; là, une femme se berce } \\
& \text { sur une galerie de bois. (50) }
\end{aligned}
$$


Significativement, le paysage familier naît de ces gestes, mais de ceux aussi, nouveaux, du sujet :

$$
\begin{aligned}
& \text { [...] Tout un été, } \\
& \text { nous avons redonné vie au bois } \\
& \text { des portes et aux lattes des murs } \\
& \text { des chambres de la maison; } \\
& \text { dans les nervures du bois, } \\
& \text { nous n'avons trouvé que la beauté } \\
& \text { de l'âge enroulé sur lui-même. (83) }
\end{aligned}
$$

Il naît aussi de l'intériorité du sujet et de ses figures métaphoriques, au sens que Pierre Nepveu a donné à ce terme dans Intérieurs du Nouveau Monde (I998) :

« Intérieurs »: ce terme doit être entendu dans un sens physique, spatial: habitations, lieux de réclusion ou de sédentarité, paysages intimes, chambres et villes; et dans un sens psychique: espace subjectif, expérience non fusionnelle d'une pensée séparée du monde et cherchant à l'appréhender, résistance à l'appel du lointain et aux élans dionysiaques. (8)

Dans le poème suivant, la cabane ou le poste d'observation/méditation fonctionne ici comme une enveloppe psychique du sujet, une interface entre son intériorité et le monde extérieur :

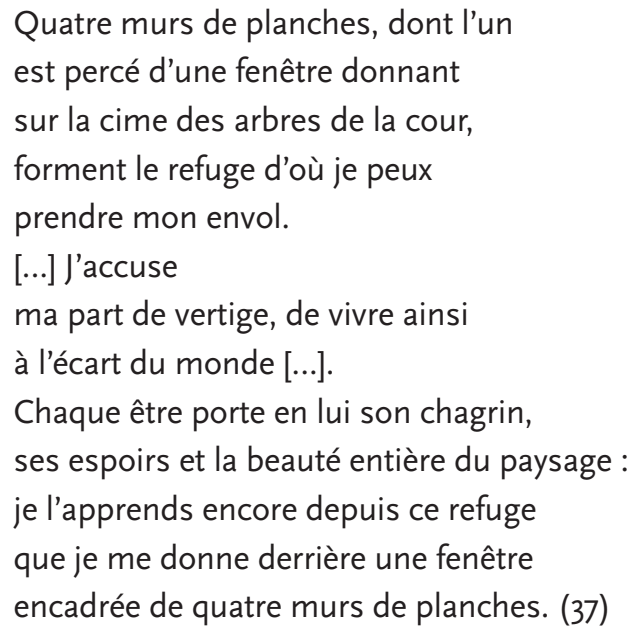

Le refuge devient lieu de rencontre avec soi-même, mais aussi avec le paysage environnant. Il n'y a pas symbiose du sujet et de l'étendue. Comme Melançon a recours à la médiation picturale, le sujet chez Paradis a besoin de ce refuge, de se placer « derrière une fenêtre » (nous soulignons), elle-même « encadrée de quatre murs de planches » (nous soulignons). Ce dispositif optique et psychique crée un effet de distanciation à l'intérieur même du paysage et permet ainsi au sujet de le mettre en mots. 
Au terme de cette réflexion qui nous a fait remonter aux sources du concept de paysage, traverser différents langages artistiques et types de paysages contemporains, il importe de souligner deux observations fondamentales. D'une part, le paysage, plus qu'un simple motif esthétique, est en passe de devenir une véritable valeur éthique qui émeut et mobilise. Plus qu’un simple cliché touristique, le paysage est en voie de devenir un lieu de rencontre, de convergence éthique et esthétique. La prégnance de ce genre dans la pratique d'Isabelle Hayeur tout comme dans un bon nombre de recueils de poèmes de grande qualité ces dernières années en atteste. Or, il est frappant de constater à quel point ces représentations paysagères demeurent fidèles à « l'esprit » premier de ce concept : conformément au contexte anthropocentriste de la Renaissance dans lequel il vit le jour, le paysage demeure subordonné au sujet qui lui préexiste. Bien souvent encore, le sujet est l'opérateur essentiel pour révéler, faire apparaître le paysage. L'homme demeure donc absolument central dans cette conception. Cela peut surprendre aujourd'hui étant donné la conscience aiguë que nous avons de la complexité du monde, de notre échelle humaine extrêmement réduite à l'échelle de la planète, sinon du cosmos, et du fait que les propres systèmes que nous mettons en place semblent nous échapper. Mais sans doute cette propension à représenter des paysages proches relève-t-elle aussi d'une autre conscience toute aussi contemporaine : pour complexe et délétère que soit devenu le monde qui nous entoure, nous sommes également de plus en plus conscients des incidences de nos choix et modes de vie sur notre environnement. Et cette conscience ne manque pas d'affleurer dans les images et les vers que nous avons parcourus, ne serait-ce que dans le choix des paysages souvent caractéristiques de l'ère postindustrielle.

\section{NOTES}

1. Voir, du côté des sciences environnementales, les travaux de Kevin McGarigal et de Samuel Cushman autour du concept de « gradient landscape » qui soulignent la variabilité, les transformations intrinsèques des paysages en dehors de toute activité humaine.

2. Anne Cauquelin (2000) constate par ailleurs que «[l]es historiens-géographes de l'Antiquité Hérodote ou Xénophon ne sont pas avares de descriptions de "lieux". Pour autant elles ne constituent pas ce que nous appelons paysages : simples conditions matérielles de l'événement, une guerre, une expédition, une légende, elles lui restent soumises. Facteurs de causalité et de signification organisant le discours et servant de cadre aux savoirs nombreux : le relief, la flore, la faune, les aménagements humains, les vestiges du passé : autant de "locations" indispensables aux récits, et qui leur sont liés. L'objet paysage ne préexiste pas à l'image qui le construit pour une visée discursive 》 (29).

3. « Life imitates Art far more than Art imitates Life » dans Le déclin du mensonge.

4. Un exemple paradigmatique en la matière serait le tableau bien connu de Friedrich, Le Voyageur contemplant une mer de nuages (1818), et du côté littéraire à la façon dont le narrateur de Jacques le fataliste de Diderot prend en compte la présence du lecteur dans son texte. Mais toute une gamme de jeux encore plus complexe peut être imaginée, et l'on pense alors au dispositif optique mis en place par Velázquez dans Les Ménines (1656). Voir la fameuse analyse que Michel Foucault (1966) donne de ce tableau dans le premier chapitre de Les mots et les choses.

5. http://isabelle-hayeur.com/bio.html.

6. http://isabelle-hayeur.com/bio.html.

7. Isabelle Hayeur, « Paysages incertains » (1998-2002), http://isabelle-hayeur.com/photos/incertains/index.html. 
8. Isabelle Hayeur, « Destinations » (2003-2004), http://isabelle-hayeur.com/photos/destinations/index.html.

9. Isabelle Hayeur, « Les bois morts », série « Paysages incertains » (1998-2002), http://isabelle-hayeur.com/photos/ incertains/bois_morts.html.

10. Isabelle Hayeur, « L'île », série « Paysages incertains » (1998-2002), http://isabelle-hayeur.com/photos/incertains/ ile.html.

11. Isabelle Hayeur, «Échappée », série « Paysages incertains » (1998-2002), http://isabelle-hayeur.com/photos/ incertains/echappee.html.

12. Isabelle Hayeur, « Les routes de sel : Lagune », série « Destinations » (2003-2004), http://www.creativetechnology. org/photo/desert-1/next?context=album\&albumld=2128459\%3AAlbum\%3A36097.

13. Isabelle Hayeur, « Les routes de sel : Oued », série « Destinations » (2003-2004), http://www.creativetechnology. org/photo/lagune-1/prev?context=album\&albumld=2128459\%3AAlbum\%3A36097.

14. Isabelle Hayeur, http://isabelle-hayeur.com/photos/destinations/index.html.

15. Isabelle Hayeur, « Maisons modèles » (2004-2007), http://isabelle-hayeur.com/photos/maisons_modeles/index. html.

16. Isabelle Hayeur, « Nuits américaines » (2004-2008), http://isabelle-hayeur.com/photos/nuit_americaine/index. html.

17. Ce terme désigne de luxueuses et récentes maisons qui paraissent hors de proportion avec le terrain (trop étroit) sur lequel elles sont bâties ou avec leur quartier (plus modeste et moins tape-à-l'œil). Disproportionnées par rapport au petit nombre d'occupants auquel elles sont destinées, elles sont souvent conçues en série et sans tenir compte de l'architecture locale. Elles se caractérisent par une esthétique kitsch et prétentieuse fondée sur le paraître au détriment d'un usage raisonné des matériaux. Les termes « Hummer House » ou « Starter Castle », tout aussi péjoratifs, peuvent se substituer à « McMansion ».

18. Isabelle Hayeur, « Jade », série « Maisons modèles », (2004-2007), http://www.creativetechnology.org/ photo/2128459:Photo:36114? context=album\&albumld=2128459\%3AAlbum\%3A36072.

19. Voir, de façon générale, l'intégralité de ce numéro de revue : « Tous banlieusards. L'hégémonie d'un idéal urbain », Marie Parent dir., Liberté, $\mathrm{n}^{\circ}$ 301, automne 2013. Voir également les travaux de Daniel Laforest sur les représentations de la banlieue en littérature : Daniel Laforest (2013), « La banlieue dans l'imaginaire québécois. Problèmes originels et avenir critique », temps zéro, $n^{\circ} 6,2013$. Également disponible en ligne : https ://tempszero.contemporain.info/ document945.

20. Isabelle Hayeur, « Excavations » (2005-2008), http://isabelle-hayeur.com/photos/excavations/index.html.

21. Isabelle Hayeur, « Underworlds" (2008-2014), http://isabelle-hayeur.com/photos/underworld/index.html.

22. http://isabelle-hayeur.com/photos/excavations/index.html.

23. Isabelle Hayeur, « Losing Ground » (2009), http://vimeo.com/4709713.

24. Isabelle Hayeur, « Private View » (2010), http://vimeo.com/17992788.

25. Isabelle Hayeur, « Déraciné » (2012), http://vimeo.com/35406035.

26. Chacun à leur manière, c'est ainsi ce qu'explorent entre autres des romans tels que La rage (1989), Cowboy (1992) et Le joueur de flûte (2001) de Louis Hamelin, Les carnets de Douglas (2007) de Christine Eddie, Les fossoyeurs (2010) d'André Lamontagne, Le ciel de Bay City (2008) de Catherine Mavrikakis, La sœur de Judith (2007) de Lise Tremblay et Dée (2002) de Michael Delisle. Du côté de l'essai, Intérieurs du Nouveau Monde (1998) et Lectures des lieux (2005) de Pierre Nepveu ont ouvert la voie, tandis que depuis quelques années, la plume véhémente de Serge Bouchard s'attaque à ce sujet sous divers angles dans Les corneilles ne sont pas les épouses des corbeaux (2005) et C'était au temps des mammouths laineux (2012). 


\section{BIBLIOGRAPHIE}

Berque, Augustin. 2000. Écoumène. Introduction à l'étude des milieux humains. Paris, Belin.

Cauquelin, Anne. 2000. L'invention du paysage. [1989]. $2^{\mathrm{e}}$ éd. Paris, Presses universitaires de France, coll. «Quadrige ».

—. 2009. Préface. Le paysage façonné. Les territoires postindustriels, l'art et l'usage de Suzanne Paquet. Québec, Presses de l'Université Laval, coll. « Recherche ».

Deguy, Michel. 2005. « Le lieu et sa nomination ». Lieux propices. L'énonciation des lieux / Le lieu d'énonciation dans les contextes francophones interculturels. Québec, Presses de l'Université Laval, coll. « Intercultures », p. 17-23.

Dictionnaire de l'Académieient française. [1694]. [En ligne], dans Dictionnaires d'autrefois. [http://artflsrvo2.uchicago.edu/ cgi-bin/dicos/pubdicoılook.pl?strippedhw=paisage] (Consulté le 9 juin 2014).

Foucault, Michel. 1966. « Les suivantes ». Les mots et les choses. Une archéologie des sciences humaines. Paris, Gallimard, coll. «NRF Bibliothèque des sciences humaines », p. 19-31.

Girardin, René-Louis. 1992. De la composition des paysages. Seyssel (France), Champ Vallon.

Harel, Simon. 2007. Espaces en perdition. Québec, Presses de l'Université Laval.

Hayeur, Isabelle. Site internet de l'artiste. [En ligne]. [http://isabelle-hayeur.com] (Consulté le 10 juin 2014).

Huston, Nancy. 1995. Pour un patriotisme de l'ambiguïté. Notes autour d'un voyage aux sources. Montréal, Fides / CÉTUQ, coll. « Les grandes conférences ».

Lapierre, René. 1995. Écrire l'Amérique. Montréal, Les Herbes rouges.

Lussier, Laurent. 2013. « Le grand collage. De Oka à Saint-Hilaire: une histoire en diagonale des banlieues montréalaises ». Liberté, n 301 (automne), p. 21-24.

McGarigal, Kevin et Samuel Cushman. 2005. «The Gradient Concept of Landscape Structure ». Issues and Perspectives in Landscape Ecology. John Wiens et Michael Moss, réd., Cambridge University Press, p. 112-119.

Melançon, Robert. 2004. Le paradis des apparences. Essai de poèmes réalistes. Montréal, Éditions du Noroît.

Merleau-Ponty, Maurice. 1966. Sens et non-sens. [1948]. $5^{\mathrm{e}}$ éd. Paris, Nagel, coll. « Pensées ».

Nepveu, Pierre. 1998. Intérieurs du Nouveau Monde: Essais sur les littératures du Québec et des Amériques. Montréal, Boréal, coll. « Papiers collés ».

2002. Lignes aériennes. Montréal, Éditions du Noroît.

Nicot, Jean. Le Thresor de la langue francoyse. [1606]. [En ligne], dans Dictionnaires d'autrefois. [http://artflsrvo2.uchicago. edu/cgi-bin/dicos/pubdicoılook.pl?strippedhw=paisage] (Consulté le 9 juin 2014).

Paradis, Claude. 2013. Carnet d'un improbable été. Montréal, Éditions du Noroît.

Roger, Alain. 1997. Court traité du paysage. Paris, Gallimard, coll. « NRF, Bibliothèque des sciences humaines ». Thibault, Louis-Jean. 2007. Reculez falaise. Montréal, Éditions du Noroît. 